\title{
Magnetophoresis of diamagnetic micro particles in a weak magnetic field
}

\author{
Gui-Ping Zhu, ${ }^{a}$ Majid Hejiazan, ${ }^{b}$ Xiaoyang Huang ${ }^{a}$ and Nam-Trung Nguyen ${ }^{b *}$,
}

Magnetic manipulation is a promising technique for lab-on-a-chip platforms. The magnetic approach can avoid problems associated with heat, surface charge, ionic concentration and $\mathrm{pH}$ level. The present paper investigates the migration of diamagnetic particles in a ferrofluid core stream that is sandwiched between two diamagnetic streams in a uniform magnetic field. The three-layer flow is expanded in a circular chamber for characterisation based on imaging of magnetic nanoparticles and fluorescent microparticles. A custom-made electromagnet generates a uniform magnetic field across the chamber. In a relatively weak uniform magnetic field, the diamagnetic particles in the ferrofluid move and spread across the chamber. Due to the magnetization gradient formed by the ferrofluid, diamagnetic particles undergo negative magnetophoresis and move towards the diamagnetic streams. The effects of magnetic field strength and concentration of diamagnetic particles are studied in details.

\section{Introduction}

Continuous-flow microfluidics manipulates particles both passively and actively. Passive methods rely purely on hydrodynamics in microchannels and the physical properties of particles to be manipulated. Depending on applications, the efficiency and throughput of passive methods are limited. Active methods require externally induced forces such as electrical, ${ }^{1-3}$ thermal $^{4}$, optical ${ }^{5-6}$, and magnetic ${ }^{7-8}$ forces. Most active concepts require a complex design for inducing the force field. Active concepts utilizing electric and optical inputs often generate unnecessary heat, which together with the required ionic concentration is often harmful for sensitive samples. Magnetic concepts can overcome the above problems and gain new functionalities in the microfluidic environment. The interaction between magnetism and fluid flow provides a truly wireless approach for microfluidic manipulation that is not affected by heat, $\mathrm{pH}$ level or ion concentration. Magnetic concepts have been employed for conductive liquid driven by Lorentz force $^{9-11}$. Magnetic particles coated with an affinity marker are commonly used for sorting diamagnetic particles such as cells. The interaction between magnetism and fluid flow leads to research areas such as ferrohydrodynamics $(\mathrm{FHD})^{12-13}$, magnetorheology (MR) ${ }^{14-15}$, and magnetophoresis (MP) ${ }^{16-17}$. Magnetophoresis has been used for various particle manipulation applications such as sorting and separation ${ }^{18-19}$, focusing ${ }^{20-21}$, assembling ${ }^{22-23}$ stirring, mixing $^{24-}$ 25 and pumping ${ }^{26-27}$.

In conventional magnetophoretic manipulation, magnetic beads are driven along a magnetic field gradient. The force acting on the magnetic particles in a diamagnetic fluid is also caused by their susceptibility mismatch. The movement of a magnetic particle towards the higher magnetic field gradient is called positive magnetophoresis. Positive magnetophoresis is suitable for separation applications, as magnetic particles are commonly used as solid support for antigens, antibodies, DNA and cells. With a functionalized coating, targeted biomolecules or cells can be labelled with magnetic particles, and subsequently trapped or sorted by an external magnetic field. ${ }^{28}$ Magnetophoresis has been used for separation of red blood cells ${ }^{29}$, isolation of progenitor cells ${ }^{30}$ and separation of breast cancer cells from human blood ${ }^{31}$. Efforts have been devoted to improve positive magnetophoretic separation such as the selection 
of magnets and flow configuration. Readers may refer to review papers on basic principles as well as the various applications of positive magnetophoresis ${ }^{32-34}$.

Most particles in analytical and biological fields exhibit diamagnetic properties. Negative magnetophoresis is the phenomenon where diamagnetic particles migrate away from the magnetic source or the higher gradient due to the magnetic buoyancy force in a paramagnetic carrier ${ }^{35}$. The magnetization of a paramagnetic carrier could be determined by the susceptibility of the liquid and the magnetic field. Ferrofluid as a paramagnetic solution with a high susceptibility suits well for the implementation of negative magnetophoresis. Ferrofluid is a stable colloidal suspension of ferromagnetic nanoparticles with diameter of less than $10 \mathrm{~nm}$. The particles are well dispersed in a diamagnetic carrier fluid. The magnetic particles are coated with a surfactant to prevent agglomeration. Since the discovery of ferrofluid in the early 1960s, this material has been used extensively in various applications. Readers may refer to Rosensweig ${ }^{36}$ for further details on ferrofluid and ferrohydrodynamics. Vékás et al. reviewed recent achievement of the synthesis of magnetic nanoparticles ${ }^{37}$. Numerous research works have been conducted to characterize ferrofluid according to particle concentration ${ }^{38}$, magnetization ${ }^{39-40}$ and viscosity ${ }^{41-42}$. Xuan and his colleagues developed a microfluidic device with embedded permanent magnets for particles manipulation ${ }^{43-44}$. The device has been used for concentrating particles ${ }^{45-46}$, separation ${ }^{47-49}$ and focusing ${ }^{50}$ of particles and cells. Mao and Koser $^{12}$ reported mixing of ferrofluid with a fluorescein buffer by using ferrohydrodynamic instabilities caused by sudden velocity variation of the flow passing by a permanent magnet. The same group demonstrated magnetic manipulation, separation and sorting of particles and cells by using ferrofluid ${ }^{19,51-52}$. Size-dependent manipulation of diamagnetic particles in ferrofluid has been realized. Separation of diamagnetic cells has been achieved with an efficiency of $100 \%{ }^{53}$ and a throughput of $10^{7}$ cells per hour ${ }^{54}$. Efforts have also been devoted to applications in particles focusing ${ }^{55-57}$. In addition to experimental investigations, analytical model was reported by Mao’s group on transport of non-magnetic particles in ferrofluids under a non-uniform magnetic field ${ }^{53,58}$. Furthermore, particles assembling ${ }^{22-23}$ have also been reported. Friedman and Yellen reviewed the underlying basic principle and models for separation, manipulation and assembly of solid diamagnetic phase using an external magnetic field. ${ }^{59}$ In all reported works on negative magnetophoresis, a non-uniform magnetic field with a high gradient is required to maximise the induced magnetic force. This magnetic field often come from a bulky permanent magnet. None of the previous works used a weak uniform magnetic field for manipulating diamagnetic particles.

We demonstrate here negative magnetophoresis of diamagnetic microparticles in a ferrofluid with a relatively weak external uniform magnetic field. Instead of using a magnetic field with high strength and gradient, our concept only requires a uniform magnetic field with strength of only few milliteslas (mT), two or three orders of magnitude lower than those of most cases reported in the literature. We also experimentally investigate the influence of the magnetic field strength and the concentration of diamagnetic microparticles. 


\section{Experimental setup and results}

We fabricated a microfluidic device that was specifically designed for the negative magnetophoresis experiments. The device has three inlets, one outlet and a circular observation chamber for better visualization of the ferrofluid and the diamagnetic particles, Figure 1(a). The circular chamber has a height of $H=50 \mu \mathrm{m}$ and a diameter of $D=1 \mathrm{~mm}$. The inlet and outlet channels have a height of $H=50 \mu \mathrm{m}$ and a width of $W=50 \mu \mathrm{m}$. The device was made of polydimethylsiloxane (PDMS), using the standard soft lithography technique. Readers may refer to Song et al. for the detailed fabrication recipe ${ }^{60}$. The PDMS device was peeled off on the mold, and access holes were created with a 0.75-mm puncher. The device was then treated with oxygen plasma and bonded to another flat PDMS piece to create a closed microfluidic device. The device was then trimmed to fit into the air gap of the electromagnet as described below.

(a)

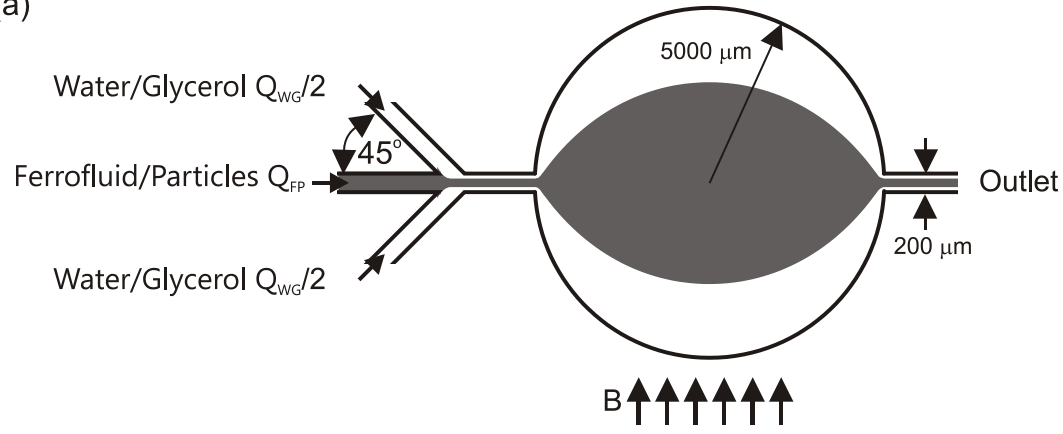

(b)

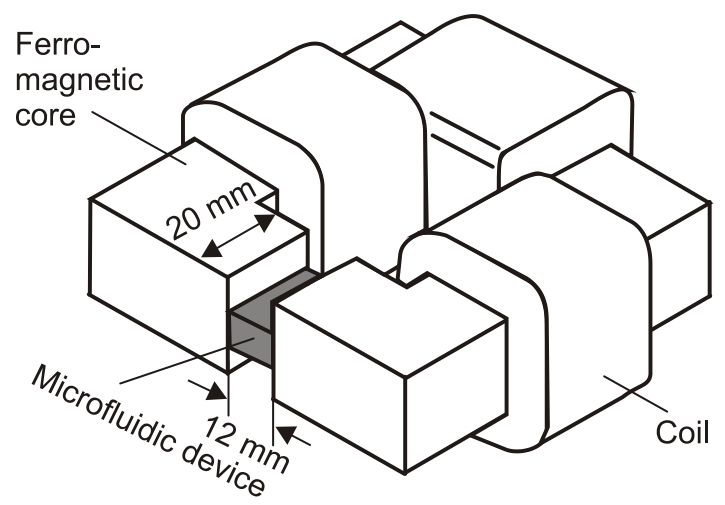

Figure 1 Experiment setup with a circular chamber located in a uniform magnetic field generated by a custom-made electromagnet: (a) Distribution of the liquid streams; (b) Custom-made electromagnet with the microfluidic device inserted in the air gap. 


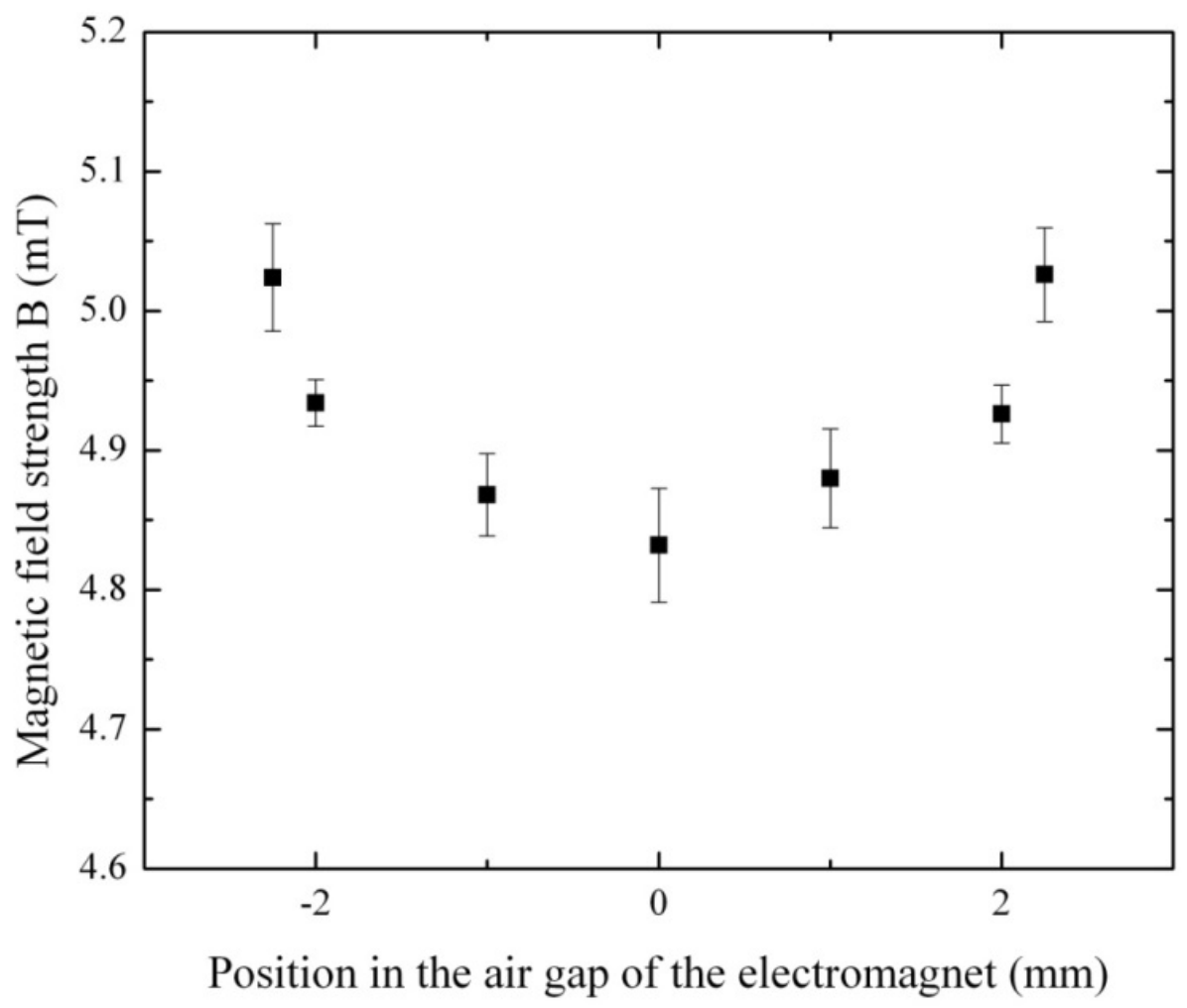

Figure 2 The measured magnetic flux density in the air gap.

Figure 1(b) depicts the experimental setup with the circular chamber inserted in the uniform field generated by a custom-made electromagnet. The electromagnet was modified from a transformer whose ferromagnetic core was cut to form an air gap of $12 \mathrm{~mm}$. The uniform magnetic field in the air gap applies across the microfluidic device. Demagnetization of the electromagnet is necessary to eliminate the residual magnetization after each experiment. Demagnetization was achieved by applying a reversed current for 5 to10 minutes using highest current value of the previous experiment. The uniformity of the magnetic field was examined with a current of 0.2 A. As the 1-mm diameter of the chamber is relatively small, the calibration was only carried out in the 4-mm around the center of the air gap. Figure 2 shows that the center of the gap has the lowest flux density. The flux density increases lightly toward the magnetic poles. As the difference of the flux density was less than $5 \%$ within the 4-mm space, the magnetic field can be assumed to be uniform in the 1-mm chamber of our experiments. The PDMS device was inserted into the air gap and therefore thermally insulated from the electromagnet.

Two precision syringe pumps (KD Scientific Inc., USA) delivered the liquids to the microfluidic device. The whole setup was placed on a Nikon (Eclipse TE2000-S) inverted microscope equipped with a digital camera (HiSense Mkll). A Laboratory DC power supply (GPS-3030D) provides the current to the electromagnet. The microfluidic device was slotted into the air gap for testing. A maximum magnetic flux density up to $53 \mathrm{mT}$ could be generated by tuning the supply current up to 2.0 A. The magnetic flux generated at different currents was measured and calibrated using a commercial gaussmeter (Hirst, GM05, UK). 
Water-based ferrofluid (EMG707, Ferrotec) was used for the core stream. The ferrofluid has saturation magnetization of $11 \mathrm{mT}$, density of $\rho_{F F}=1.1 \times 10^{3} \mathrm{~kg} \mathrm{~m}^{-3}$, viscosity of $\eta_{F F}=5 \mathrm{mPa} \cdot \mathrm{s}\left(\right.$ at $\left.27^{\circ}\right)$, magnetic particle concentration of $2 \% \mathrm{vol}$, and an initial susceptibility of $\chi_{F F}=0.36$. The magnetization characteristics of this ferrofluid were described in our previous work. ${ }^{61}$ Green fluorescent diamagnetic polymer microparticles with a diameter of $1.0 \mu \mathrm{m}$ (Duke Scientific, 1\% Solids) were mixed with DI water and the ferrofluid at different concentrations. Solutions with four different concentrations were used in the experiment, and termed respectively as sample I, II, III and IV, Table 1.

Table 1 Different core stream samples used in the experiments.

\begin{tabular}{lc}
\hline Samples & Composition of the core stream \\
\hline I & $50 \mathrm{wt} \%$ DI water and $50 \mathrm{wt} \%$ Ferrofluid \\
II & $25 \mathrm{wt} \%$ DI water and $25 \mathrm{wt} \%$ microspheres suspension and $50 \mathrm{wt} \%$ Ferrofluid \\
III & $12.5 \mathrm{wt} \%$ DI water and $37.5 \mathrm{wt} \%$ microspheres suspension and $50 \mathrm{wt} \%$ Ferrofluid \\
IV & $50 \mathrm{wt} \%$ microspheres suspension and $50 \mathrm{wt} \%$ Ferrofluid \\
\hline
\end{tabular}

The diamagnetic liquid is a mixture of DI water and glycerol (16371, Affymetrix). Glycerol has a density of $\rho_{G}=1.26 \times 10^{3} \mathrm{~kg} \mathrm{~m}^{-3}$, and a viscosity of $\eta_{G}=1410 \mathrm{mPa} \cdot \mathrm{s}$ at $20^{\circ} \mathrm{C}$. In order to obtain a viscosity comparable to the ferrofluid, water/glycerol mixture was used with a viscosity of $5 \mathrm{mPa} \cdot \mathrm{s}$ at $25^{\circ} \mathrm{C}$ (50 wt\% DI water and $50 \mathrm{wt} \%$ glycerol). The corresponding density of the liquid at $25^{\circ} \mathrm{C}$ is $1.13 \times 10^{3} \mathrm{~kg} \mathrm{~m}^{-3}$. In the absence of a magnetic field, a clear interface is formed between the ferrofluid/particle (FP) stream and the DI water/glycerol (WG) stream as shown in Figure 1(b). At a temperature of $T=300 \mathrm{~K}$, the diffusion coefficient of the magnetic nanoparticles $\left(d_{\mathrm{p}}=10 \mathrm{~nm}\right)$ into the water/glycerol mixture is estimated by Einstein's model as $D=k_{\mathrm{B}} T /\left(3 \pi \eta_{W G} d_{\mathrm{p}}\right)=8.79 \times 10^{-12} \mathrm{~m}^{2} \mathrm{~s}^{-1}$.

The experiments were carried out with FP stream acting as the core that is sandwiched between two WG streams. Different flow rate ratios were used in the experiment to study both the migration of magnetic nanoparticles as well as negative magnetophoresis of diamagnetic microparticles. The FP suspensions were delivered into the middle inlet at a constant flow rate of $0.5 \mathrm{ml} \mathrm{h}$. The WG solution served as the cladding stream with three different flow rates of 0.25, 0.5 and $0.75 \mathrm{ml} \mathrm{h}^{-1}$. Based on the properties of the WG solution, the Reynolds number range was determined as $\mathrm{Re}=\rho_{W G} U D_{\mathrm{h}} / \eta_{W G}=0.896 \times 10^{-1}$ to $1.49 \times 10^{-1}$. Using the estimated diffusion coefficient of magnetic nanoparticles towards the WG solution, the Peclet number range is calculated as $P e=W U / D=4.74 \times 10^{5}$ to $7.90 \times 10^{5}$. The small magnitude of Reynolds number implies a laminar flow inside the chamber. Inertial effects such as recirculation at the sudden expansion are negligible. The large Peclet number means that diffusion is negligible. 


\section{Results and Discussions}

For a fixed weight ratio in the FP suspension, the stable migration of the streams without a magnetic field was investigated for the effect of flow rate ratio $Q_{W G} / Q_{F P}$. The recorded images were processed, and the intensity profile across the chamber was plotted. As the images were recorded with both through light and an epi-fluorescent filter (Nikon B-2A, excitation filter for 450-490 nm, dichroic mirror for $505 \mathrm{~nm}$ and an emission filter for $520 \mathrm{~nm}$ ), the migration of non-fluorescent magnetic nanoparticles and negative magnetophoresis of fluorescent diamagnetic microparticles can be distinguished. Without diamagnetic microparticles, the behavior of magnetic particles under a uniform magnetic field was systematically studied and reported previously. ${ }^{8}$ The continuum models are still applicable as the diamagnetic microparticles are at least 2 order of magnitudes larger than the magnetic nanoparticles.
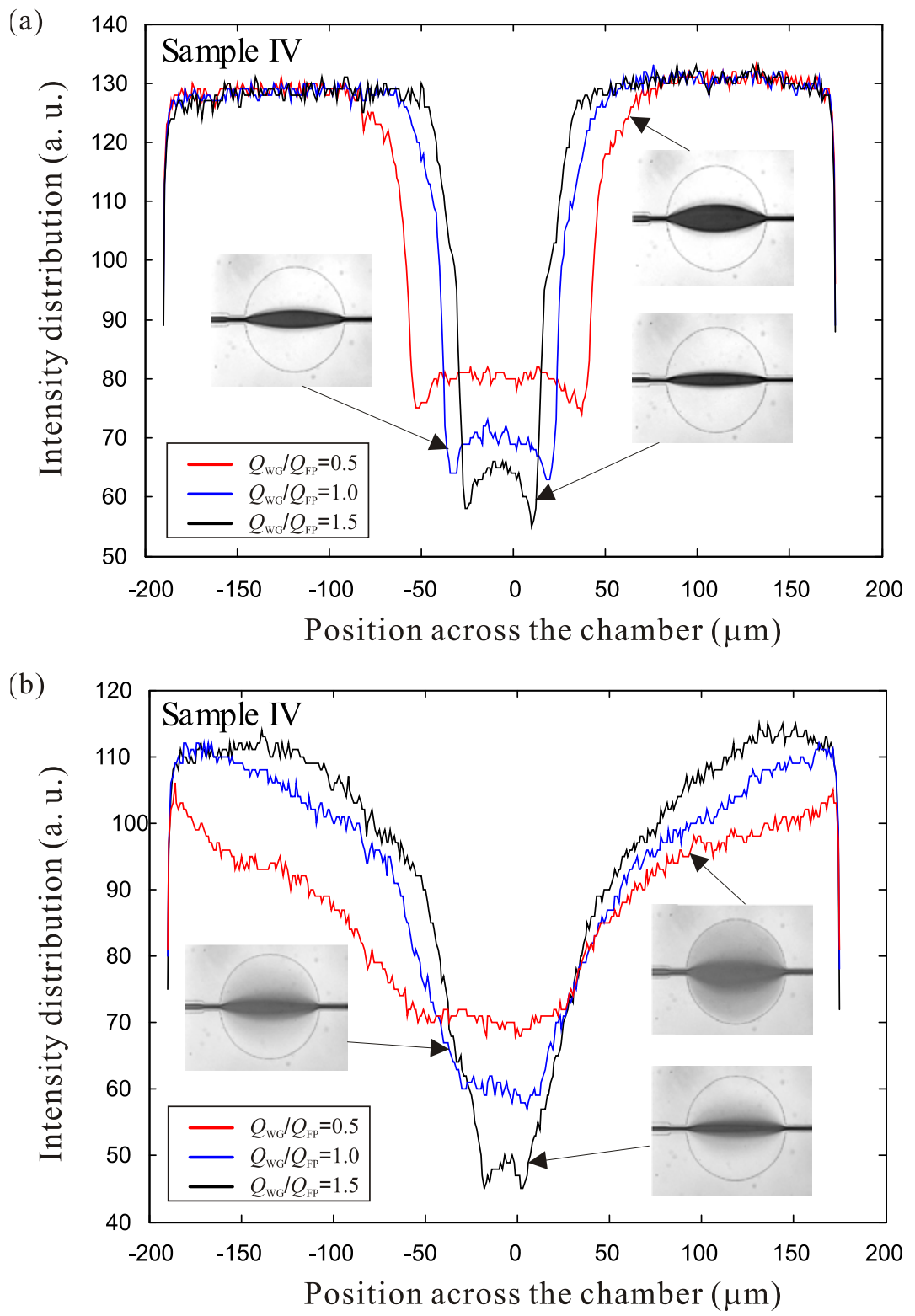

Figure 3 The spreading of non-fluorescent ferrofluid presented by intensity profile across the circular chamber perpendicular to the flow direction: (a) No magnetic field; (b) With a magnetic field of $32 \mathrm{mT}$. 
Figure 3 shows that the ferrofluid spreads towards the diamagnetic solution, even in the relatively weak magnetic field generated across the circular chamber. With fixed liquid properties (sample II), the flow rate ratio between cladding (WG) and core (FP) streams determines the initial distribution of the liquids inside the circular chamber, Figure 3(a). A larger flow rate ratio leads to a higher gradient in the final distribution of magnetic particles, Figure 3(b). The role of fluid flow in this behavior is similar to that in the convective/diffusive transport. The enhanced particle migration through magnetophoresis could possibly be characterized by an effective diffusion coefficient.

The effect of diamagnetic microparticles inside the ferrofluid on the migration performance was subsequently investigated. As indicated in Table 1, the concentration of magnetic nanoparticles is the same for all samples. The flow rate ratio between the cladding and core streams was fixed at 0.5 .

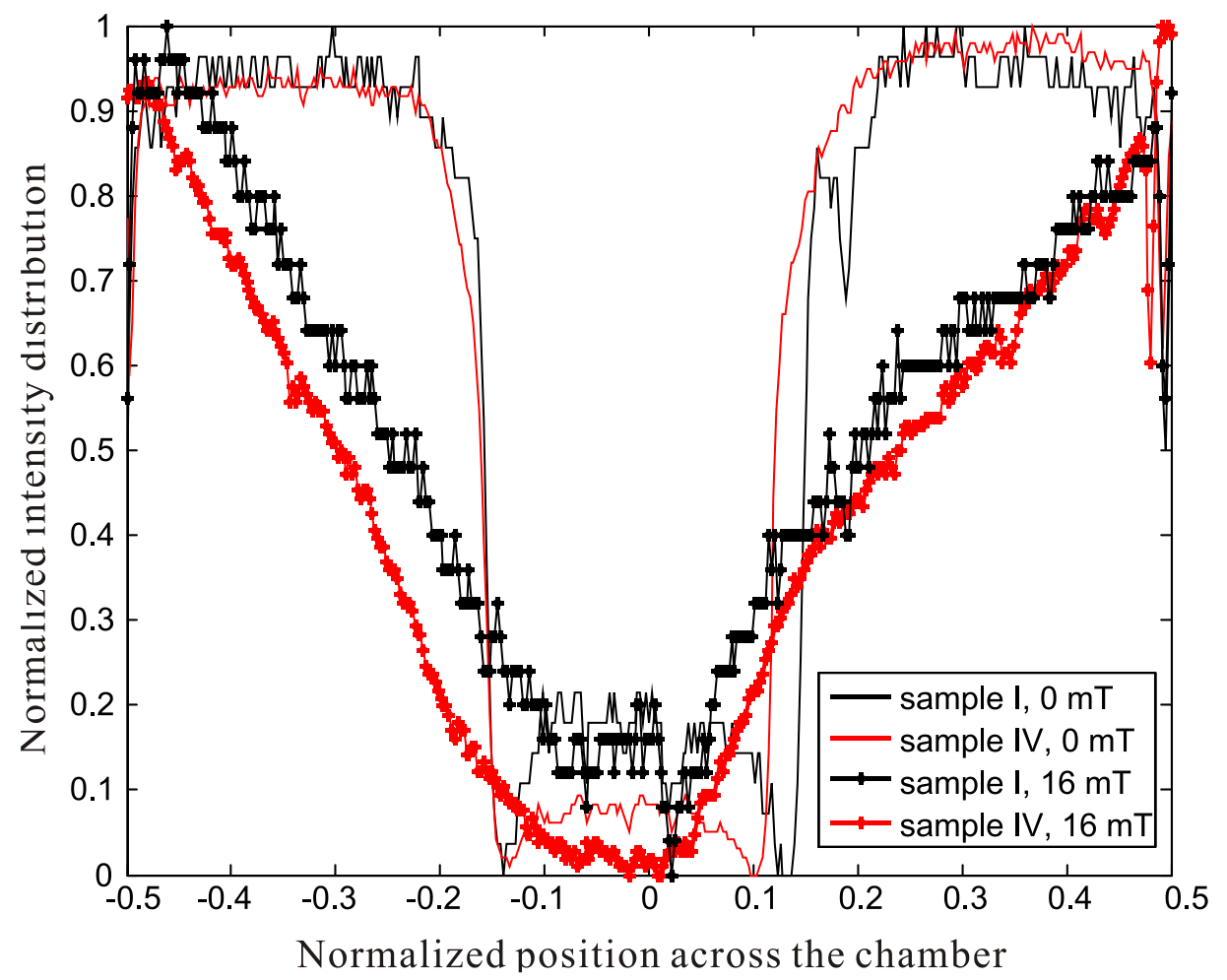

Figure 4 Comparison of the migration of non-fluorescent magnetic nanoparticles using sample I (without diamagnetic particles) and sample IV (with diamagnetic particles) at a magnetic flux density of $16 \mathrm{mT}$. The presence of diamagnetic particles improves the migration of magnetic nanoparticles.

Figure 4 shows the migration of magnetic particles in samples I and IV. The data were plotted with normalized intensity and normalized position along the chamber. Without the magnetic field, both samples show a similar migration behavior, which is determined by hydrodynamic migration only. However, as the magnetic field strength increases, the presence of diamagnetic microparticles contributes to a stronger migration of the magnetic nanoparticles, Figure 4. Besides the magnetic force, an additional hydrodynamic force enhances the migration of the magnetic nanoparticles: 


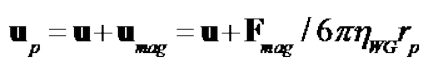

where $\mathbf{u}_{p}$ is the velocity of the particles with radius $r_{\mathrm{p}}, \mathbf{u}$ is the flow field, $r_{p}$ is the radius of the magnetic nanoparticles, and $\mathbf{F}_{\text {mag }}$ is the magnetic force on each magnetic nanoparticle, $\eta_{\mathrm{WG}}$ is the viscosity of the water/glycerol mixture. The secondary flow field induced by the motion of the diamagnetic microparticles contributes to the improved migration.

The redistribution of magnetic nanoparticles induces the migration of the FP stream leading to a concentration gradient of magnetic nanoparticles away from the center of the chamber. This concentration gradient in turn leads to an increasing magnetization gradient. Driven by the negative magnetophoretic force, diamagnetic microparticles move towards the lower magnetization gradient, e. g. in the same direction as the magnetic nanoparticles. For the same magnetic field strength, the size of diamagnetic microparticles and the concentration of magnetic nanoparticles determine the magnitude of the magnetophoretic force on the microparticles:

$$
\mathbf{F}_{d i m}=V_{p} \mu_{0} \nabla \chi \mid \mathbf{H}^{2} C_{m}
$$

where $V_{p}$ and $C_{m}$ are the volume of diamagnetic microparticles and the concentration of magnetic nanoparticles (volume fraction); $\mathbf{H}, \chi$ and $\mu_{0}$ are the volume of the magnetic nanoparticle, magnetic field strength, susceptibility of liquid and permeability of free space which has a constant value of $4 \pi \times 10^{-7} \mathrm{NA}^{-2}$. To distinguish the microparticles from the nanoparticles, the same recording was made for the results shown in Figure 3, but with the epi-fluorescent filter. Figure 5 shows the distribution of diamagnetic microparticles with a magnetic field strength ranging from $0 \mathrm{mT}$ [Figure 5(a)] to $32 \mathrm{mT}$ [Figure 5(b)]. The diamagnetic microparticles move towards the WG stream at a relatively low flux density of $32 \mathrm{mT}$. Following the behavior of magnetic nanoparticles, a higher flow rate ratio leads to a larger concentration gradient of the microparticles indicating the role of convective transport. 

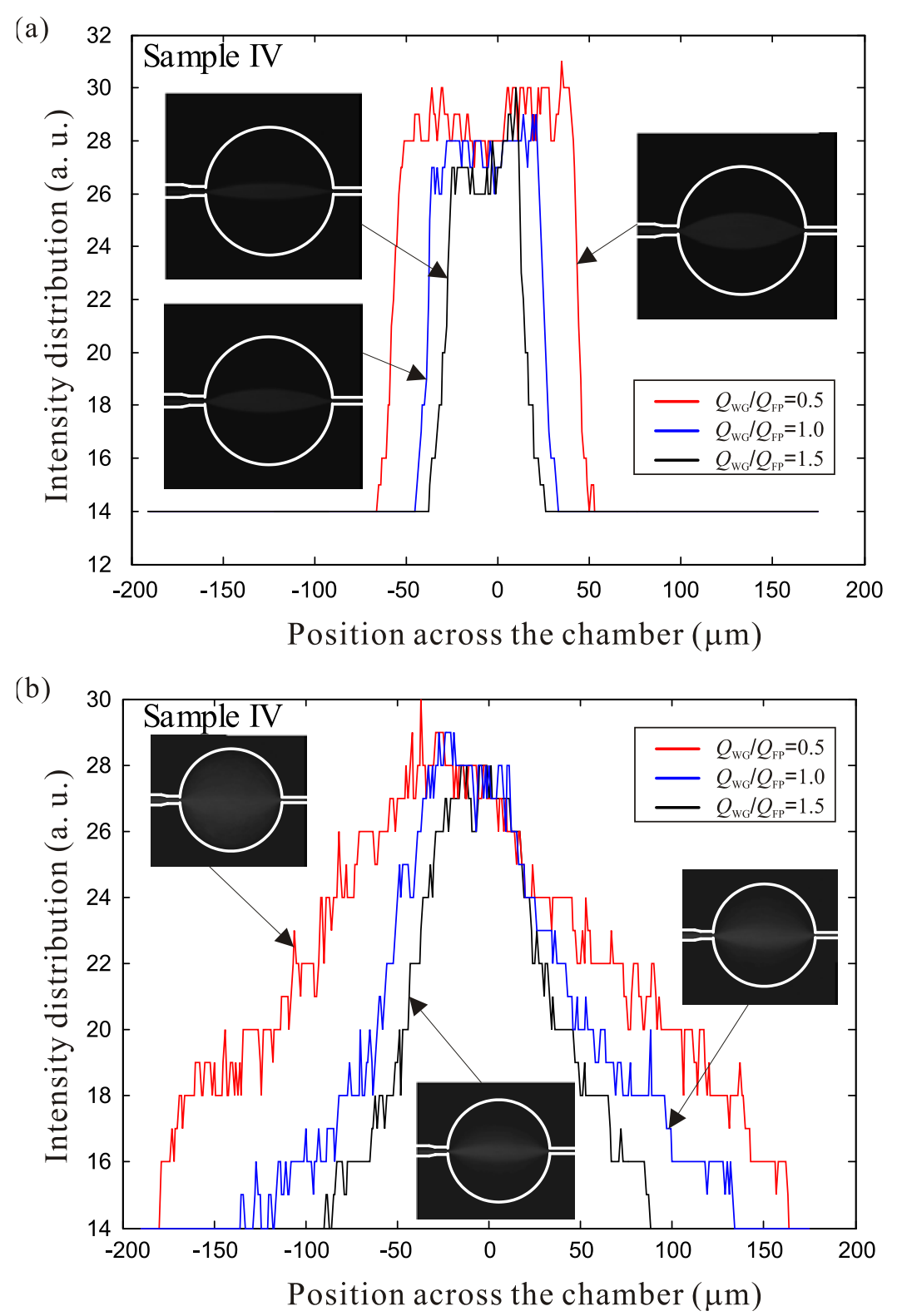

Figure 5 Negative magnetophoresis of fluorescent diamagnetic microparticles presented by their intensity profile across the circular chamber: (a) No magnetic field, the initial core width is determined by the flow rate ratio; (b) With a magnetic flux density of $32 \mathrm{mT}$. 


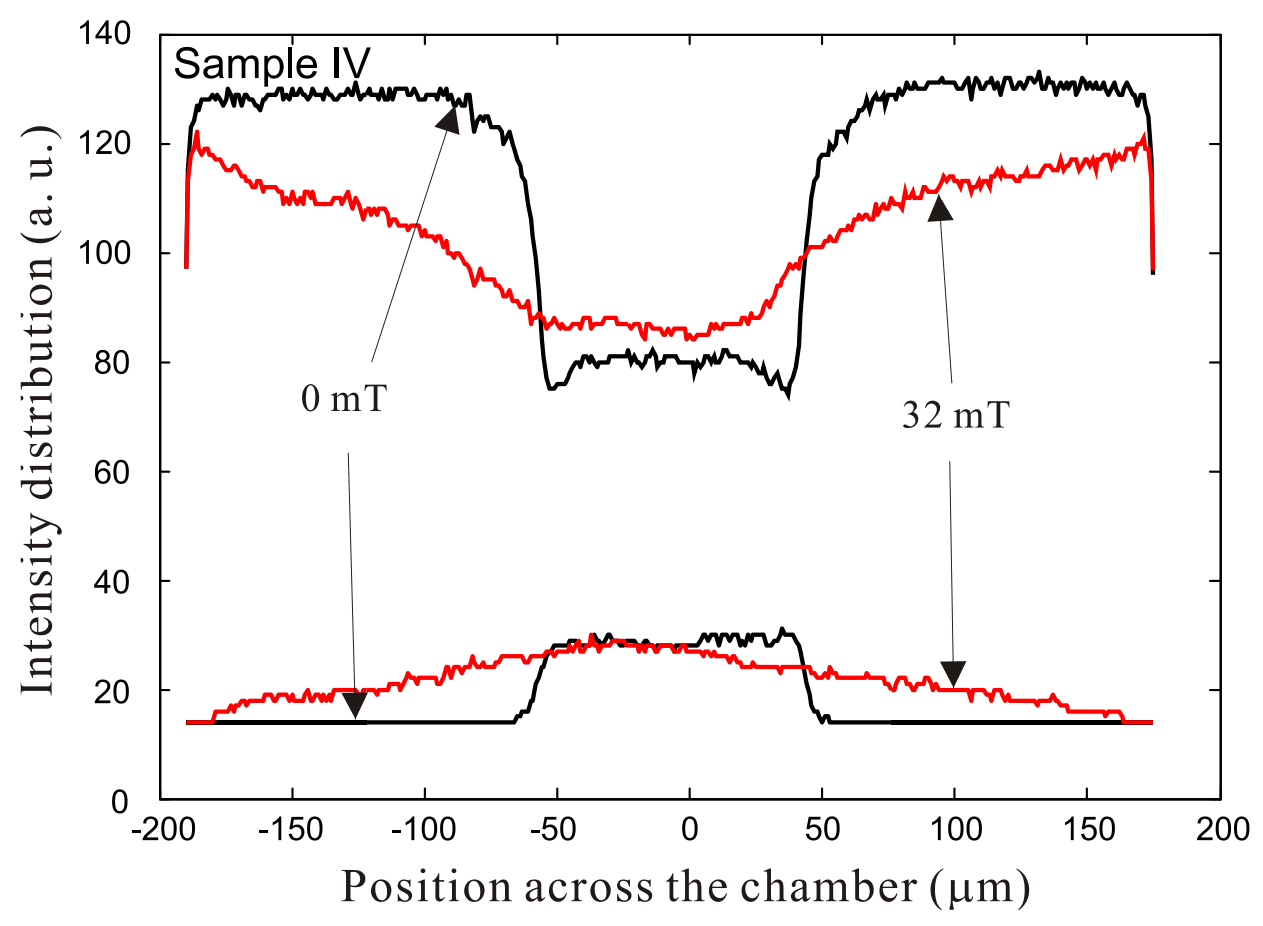

Figure 6 Migration of non-fluorescent magnetic nanoparticles (top) and fluorescent diamagnetic microparticles (bottom) with magnetic flux densities of 0 and $32 \mathrm{mT}$.

To confirm that the migration behavior of diamagnetic microparticles follows that of magnetic nanoparticles, their distribution are plotted in the same graph. Figure 6 shows the distribution of diamagnetic microparticles and magnetic nanoparticles across the chamber at $0 \mathrm{mT}$ and $32 \mathrm{mT}$, respectively. The flow rate ratio between the cladding and core stream $Q_{W G} / Q_{F P}$ was fixed at 0.5 . The intensity profile confirms that the diamagnetic microparticles migrate together with magnetic nanoparticles. However, since the magnetic forces on the magnetic and diamagnetic particles differ, their migration velocities may be different. Following, various magnetic flux densities are employed to study the field dependence of the observed phenomenon. Different concentrations of microparticles were tested to understand the role of particle concentrations.

Using sample II as the core stream, the distribution of diamagnetic microparticles across the circular chamber was examined. The motion of diamagnetic microparticles was then characterized by normalizing fluorescent intensity distribution at various magnetic field strengths, Figure 7. The flow rate ratio $Q_{W G} / Q_{F P}$ between the cladding and the core stream was also fixed at 0.5. Initially, the distribution of fluorescent diamagnetic microparticles shows a sharp interface between the two fluids. With a relatively small magnetic field, the microparticles start to move towards the WG stream. The magnetic field strength was then slowly tuned up to promote the motion of the diamagnetic microparticles. Magnetic flux densities beyond $10 \mathrm{mT}$ show a saturated state. No significant enhancement of negative magnetophoresis was observed. This agrees well with the saturation magnetization of $11 \mathrm{mT}$ of the ferrofluid in use. 


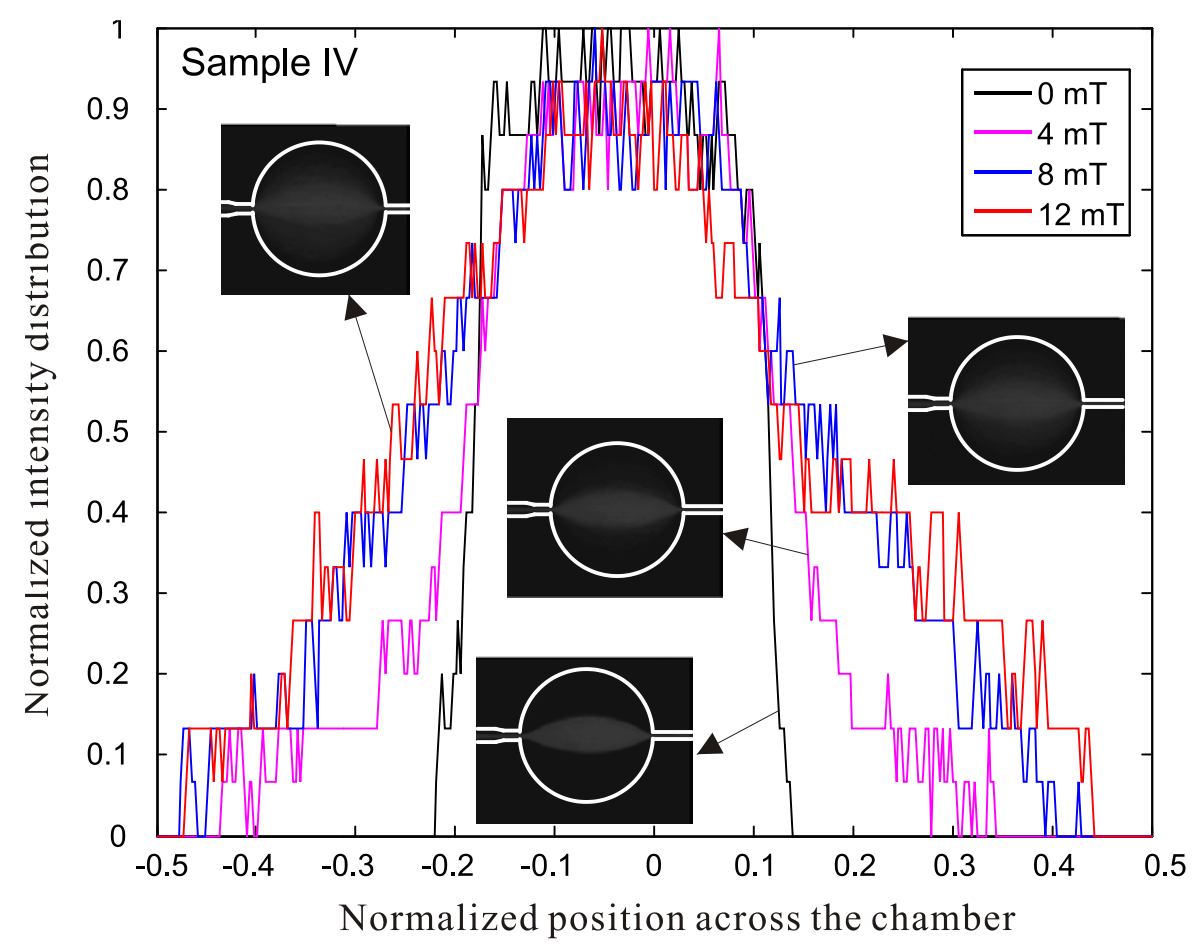

Figure 7 Normalized intensity distribution across the chamber at various magnetic flux densities (Sample IV). A stronger field leads to stronger migration. The distribution remains unchanged with flux density beyond $10 \mathrm{mT}$, corresponding to the saturated magnetization of the magnetic nanoparticles.

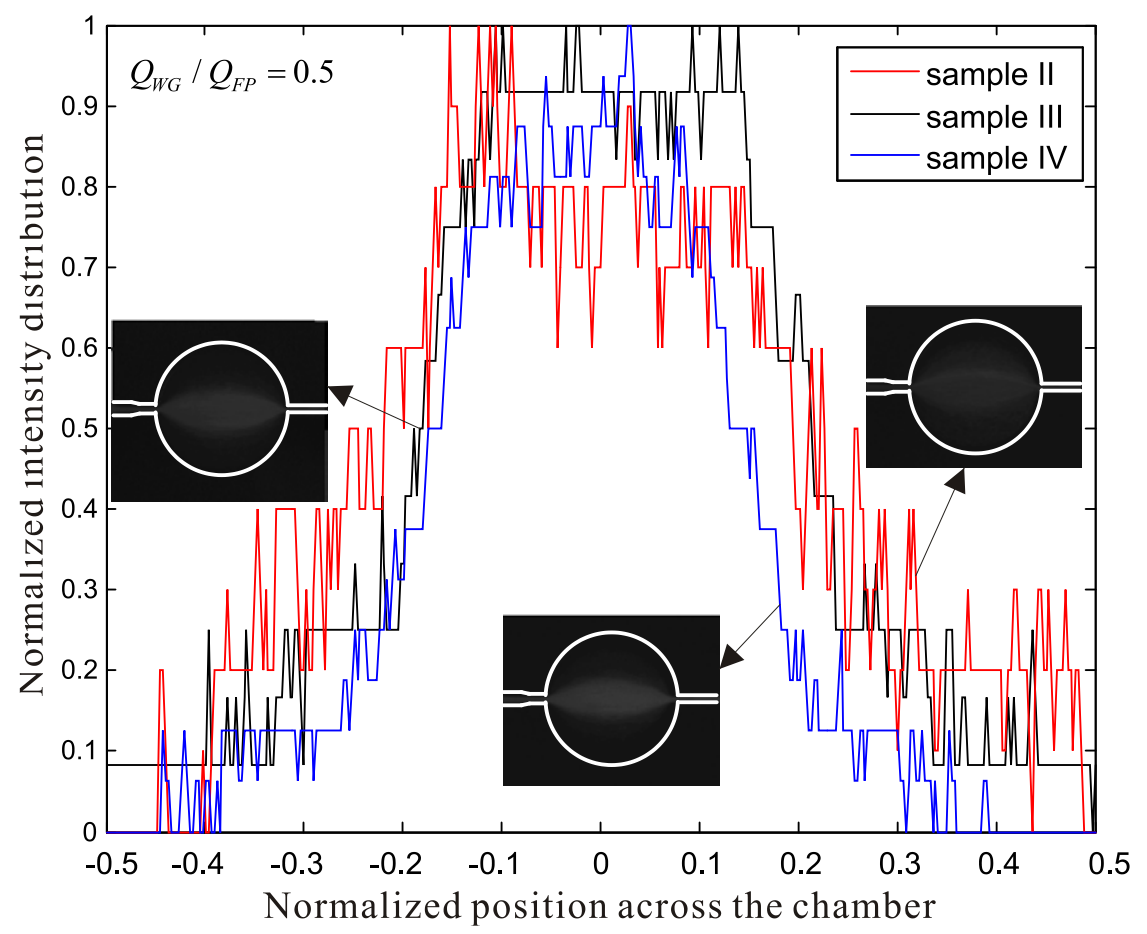

Figure 8 Negative magnetophoresis of diamagnetic microparticles with different particle concentrations in the core stream. A higher concentration of diamagnetic microparticles leads to a weaker migration. 
Table 1 indicates that samples II, III and IV have the same weight percentage of ferrofluid. Because of the initial uniform distribution, the concentrations of magnetic particles are expected to be the same in all samples. With a fixed flow rate ratio and magnetic field strength, we can then examine the effect of the concentration of diamagnetic microparticles on negative magnetophoresis. The flow rate ratio $Q_{W G} / Q_{F P}$ between the cladding and core stream was again fixed at 0.5 . The magnetic flux density was set at $4 \mathrm{mT}$. Figure 8 shows negative magnetophoresis of diamagnetic microparticles with different concentrations of diamagnetic microparticles in the core stream. Magnetophoretic migration is weaker at a higher concentration of the microparticles. A strong interaction between microparticles is expected at a higher concentration. This interaction may affect and limit the migration of the microparticles.

\section{Conclusions}

Magnetophoretic force was utilized to achieve migration of diamagnetic microparticles in a surrounding ferrofluid under a weak uniform magnetic field. A three-stream flow was generated in a circular chamber for better visualization of the migration effect. The cladding diamagnetic streams consist of DI water and glycerol. The core stream consists of ferrofluid and diamagnetic microparticles. A uniform magnetic field was generated using a custommade electromagnet. Upon activating the magnetic field across the chamber, the magnetic nanoparticles in ferrofluid migrate towards the diamagnetic cladding stream. As result, diamagnetic microparticles also move towards the same direction due to negative magnetophoresis caused by the generated magnetization gradient. The migration of microparticles and magnetic nanoparticles were studied for different flow rate ratios between cladding and core streams. Up to the saturation limit, a stronger magnetic field leads to stronger migration of both magnetic nanoparticles and diamagnetic microparticles. A higher flow rate ratio leads to a higher a concentration gradient of both particle types, indicating the limiting role of convective transport. And finally, a higher concentration of diamagnetic microparticles leads to weaker migration due to their strong interaction. 


\section{Acknowledgements}

NTN acknowledges funding support from Griffith University through a start-up grant and a grant of Griffith University Research Infrastructure Program (GURIP). MH is supported through a Griffith University International Postgraduate Research Scholarships (GUIPRS).

\section{Notes and references}

a School of Mechanical and Aerospace Engineering, Nanyang Technological University, 50 Nanyang Avenue, Singapore, Singapore 639798.

${ }^{b}$ Queensland Micro and Nanotechnology Centre, Griffith University, Brisbane, 4111, Australia.

* Corresponding author, email: nam-trung.nguyen@griffith.edu.au

Electronic Supplementary Information (ESI) available: [details of any supplementary information available should be included here]. See DOI: 10.1039/b000000x/

1. P. K. Wong, T.-H. Wang, J. H. Deval and C.-M. Ho, Mechatronics, IEEE/ASME Transactions on, 2004, 9, 366-376.

2. O. D. Velev and K. H. Bhatt, Soft Matter, 2006, 2, 738-750.

3. J. Voldman, Annu. Rev. Biomed. Eng., 2006, 8, 425-454.

4. R. Piazza, Soft Matter, 2008, 4, 1740-1744.

5. A. Jonáš and P. Zemanek, Electrophoresis, 2008, 29, 4813-4851.

6. A. Ashkin, Proceedings of the National Academy of Sciences, 1997, 94, 4853-4860.

7. N.-T. Nguyen, G. Zhu, Y.-C. Chua, V.-N. Phan and S.-H. Tan, Langmuir, 2010, 26, 12553-12559.

8. G.-P. Zhu and N.-T. Nguyen, Lab on a Chip, 2012, 12, 4772-4780.

9. J. Zhong, M. Yi and H. H. Bau, Sensors and Actuators A: Physical, 2002, 96, 59-66.

10.D. J. Sadler, R. Changrani, C. F. Chou, D. Zindel, J. Burdon and F. Zenhausern, in Micromachining and Microfabrication, International Society for Optics and Photonics, 2001, pp. 162-170.

11. M. Yi, S. Qian and H. H. Bau, J. Fluid Mech., 2002, 468, 153-177.

12. L. Mao and H. Koser, in Solid-State Sensors, Actuators and Microsystems Conference, 2007. TRANSDUCERS 2007. International, 2007, pp. 1829-1832.

13. N.-T. Nguyen and M.-F. Chai, Micro and Nanosystems, 2009, 1, 17-21.

14. J. Liu, E. Lawrence, A. Wu, M. Ivey, G. Flores, K. Javier, J. Bibette and J. Richard, Physical review letters, 1995, 74, 2828.

15. P. S. Doyle, J. Bibette, A. Bancaud and J.-L. Viovy, Science, 2002, 295, 2237-2237.

16. M. Tondra, M. Granger, R. Fuerst, M. Porter, C. Nordman, J. Taylor and S. Akou, Magnetics, IEEE Transactions on, 2001, 37, 2621-2623.

17. M. A. Gijs, Microfluidics and Nanofluidics, 2004, 1, 22-40.

18. S. Miltenyi, W. Müller, W. Weichel and A. Radbruch, Cytometry, 1990, 11, 231-238.

19. A. R. Kose, B. Fischer, L. Mao and H. Koser, Proceedings of the National Academy of Sciences, 2009, 106, 2147821483. 
20. L. Liang and X. Xuan, Microfluidics and Nanofluidics, 2012, 13, 637-643.

21. J. Shi, X. Mao, D. Ahmed, A. Colletti and T. J. Huang, Lab Chip, 2008, 8, 221-223.

22. B. Yellen, G. Friedman and A. Feinerman, Journal of applied physics, 2003, 93, 7331-7333.

23. B. B. Yellen and G. Friedman, Langmuir, 2004, 20, 2553-2559.

24. J. Berthier and F. Ricoul, in Proc, 2002 MSM Conference, 2002, pp. 22-25.

25. C. Y. Wen, C. P. Yeh, C. H. Tsai and L. M. Fu, Electrophoresis, 2009, 30, 4179-4186.

26. A. Hatch, A. E. Kamholz, G. Holman, P. Yager and K. F. Böhringer, Microelectromechanical Systems, Journal of, 2001, 10, 215-221.

27.C. Yamahata, M. Chastellain, V. K. Parashar, A. Petri, H. Hofmann and M. A. Gijs, Microelectromechanical Systems, Journal of, 2005, 14, 96-102.

28. R. Molday, S. Yen and A. Rembaum, 1977.

29. M. Zborowski, G. R. Ostera, L. R. Moore, S. Milliron, J. J. Chalmers and A. N. Schechter, Biophysical journal, 2003, 84, 2638-2645.

30. L. R. Moore, A. R. Rodriguez, P. S. Williams, K. McCloskey, B. J. Bolwell, M. Nakamura, J. J. Chalmers and M. Zborowski, Journal of magnetism and magnetic materials, 2001, 225, 277-284.

31. M. Nakamura, K. Decker, J. Chosy, K. Comella, K. Melnik, L. Moore, L. C. Lasky, M. Zborowski and J. J. Chalmers, Biotechnology progress, 2001, 17, 1145-1155.

32. N. Pamme, Lab on a Chip, 2006, 6, 24-38.

33. M. A. Gijs, F. Lacharme and U. Lehmann, Chemical reviews, 2009, 110, 1518-1563.

34. N.-T. Nguyen, Microfluidics and Nanofluidics, 2012, 12, 1-16.

35. R. E. Rosensweig, AIAA Journal, 1966, 4, 1751-1758.

36. R. E. Rosensweig, Ferrohydrodynamics, Cambridge University Press, New York, 1985.

37. L. Vékás, D. Bica and M. V. Avdeev, China Particuology, 2007, 5, 43-49.

38. R. Gerber, M. Takayasu and F. Friedlaender, Magnetics, IEEE Transactions on, 1983, 19, 2115-2117.

39. K. Morozov and A. Lebedev, J. Magn. Magn. Mater., 1990, 85, 51-53.

40. M. S. Wertheim, The Journal of Chemical Physics, 1971, 55, 4291-4298.

41. S. Odenbach, Applied Rheology, 2000, 10, 178-184.

42. J. P. McTague, The Journal of Chemical Physics, 1969, 51, 133-136.

43. L. Liang, J. Zhu and X. Xuan, Biomicrofluidics, 2011, 5, 034110.

44. J. Zhu, L. Liang and X. Xuan, Microfluid. Nanofluid., 2012, 65-73.

45. J. Zeng, C. Chen, P. Vedantam, T.-R. Tzeng and X. Xuan, Microfluid. Nanofluid., 2013, 15, 49-55.

46. J. J. Wilbanks, G. Kiessling, J. Zeng, C. Zhang, T.-R. Tzeng and X. Xuan, J. Appl. Phys., 2014, 115, -.

47. L. Liang, C. Zhang and X. Xuan, Appl. Phys. Lett., 2013, 102, 234101.

48. J. Zeng, Y. Deng, P. Vedantam, T.-R. Tzeng and X. Xuan, J. Magn. Magn. Mater., 2013, 346, 118-123.

49. L. Liang and X. Xuan, Biomicrofluidics, 2012, 6, 044106.

50. J. Zeng, C. Chen, P. Vedantam, V. Brown, T.-R. J. Tzeng and X. Xuan, J. Micromech. Microeng., 2012, 22, 105018.

51. T. Zhu, R. Cheng, Y. Liu, J. He and L. Mao, Microfluidics and Nanofluidics, 2014, 1-10.

52. T. Zhu, F. Marrero and L. Mao, Microfluid. Nanofluid., 2010, 9, 1003-1009.

53. T. Zhu, D. J. Lichlyter, M. A. Haidekker and L. Mao, Microfluid. Nanofluid., 2011, 10, 1233-1245. 
54. T. Zhu, R. Cheng, S. A. Lee, E. Rajaraman, M. A. Eiteman, T. D. Querec, E. R. Unger and L. Mao, Microfluid. Nanofluid., 2012, 645-654.

55. T. Zhu, R. Cheng and L. Mao, Microfluid. Nanofluid., 2011, 11, 695-701.

56. T. Zhu, R. Cheng and L. Mao, IEEE, 2011, pp. 1280-1283.

57. L. Liang and X. Xuan, Microfluid. Nanofluid., 2012, 1-7.

58. R. Cheng, T. Zhu and L. Mao, Microfluid. Nanofluid., 2014, 16, 1143-1154.

59. G. Friedman and B. Yellen, Current opinion in colloid \& interface science, 2005, 10, 158-166.

60. C. Song, N. T. Nguyen, S. H. Tan and A. K. Asundi, Lab Chip, 2009, 9, 1178-1184.

61. G. Zhu, N. T. Nguyen, R. Ramanujan and H. Xiaoyang, Langmuir, 2011. 